\title{
The Impact of Aberration-Corrected Transmission Electron Microscopy on Catalysis Investigations
}

\author{
P. Specht, ${ }^{*}$ R. Gulotty Jr., ${ }^{* *}$ D. Barton, ${ }^{* *}$ R. Cieslinski, ${ }^{* *}$ S. Rozeveld, ${ }^{* *}$ J. Kang, ${ }^{* *}$ C. Kisielowski*** \\ * University of California, Dept. of Materials Science and Engineering, Berkeley CA94720 \\ ** Dow Chemical Company, Midland, MI 48642 \\ *** National Center for Electron Microscopy, Lawrence Berkeley National Laboratory, One Cyclotron \\ Rd., Berkeley CA 94720
}

The new generation of aberration-corrected electron microscopes largely improved on phase contrast imaging, now enabling single atom sensitivity [1,2]. The first instrument within DoE's TEAM Project [3], TEAM0.5, was used to image industrial catalyst materials, among them $\mathrm{Rh} / \mathrm{Mo}$ on alumina support, which can be used for $\mathrm{CO}$ conversion to optimize fuel efficiency. The rhodium on alumina catalyst is known for many years (see for example [4]); molybdenum additions are used as promoters. The TEAM0.5 instrument is equipped with two Cs correctors, a high brightness gun, monochromator and stabilized electronics / mechanics. It was operated at $300 \mathrm{kV}, 80 \mathrm{kV}$ and $50 \mathrm{kV}$. The reduction of acceleration voltage led to decreased electron beam - sample interaction, visible as a slower surface motion of atoms. However, even at the lowest operating voltage of $50 \mathrm{kV}$ beam induced atom motion can still be observed. The information transfer at those low acceleration voltage images is still better than $1 \AA$ as can be seen in Fig. 1. At $80 \mathrm{kV}$ experiments were performed to study the time evolution of surface structures on a single atom level. In these experiments sub-Angstrom resolution was maintained. In figure 2 we show atom re-arrangements on the surface of a Rh particle over a time period of 17 seconds. Quantitative data analysis clarifies that the observed particle is 2-3 atoms thick and that single atom motion occurs on the particle surfaces. Similar results were observed studying Mo particles. In general, we find that the chemical identity of single atoms can be deduced from their contrast if the focus of an image is known to an accuracy of $\sim 1 \mathrm{~nm}$. Even single light atoms such as carbon, nitrogen, or oxygen can be observed adjacent to columns of heavy atoms. As a result our approach is very suitable to study details of catalytic processes dynamically. [5]

References:

1. C. Kisielowski et al., Microscopy and Microanalysis 14454-462 (2008)

2. J. Meyer, C. Kisielowski, R. Erni, M. D. Rossell, M. F. Crommie, A. Zettl, Nano Lett., 2008, 8, 3582-3586

3. http://ncem.lbl.gov/TEAM-project/index.html

4. M. Heemeier et al., Catalysis Letters 68, 19 (2000)

5. The TEAM project is supported by the Department of Energy, Office of Science, and Basic Energy Sciences. 


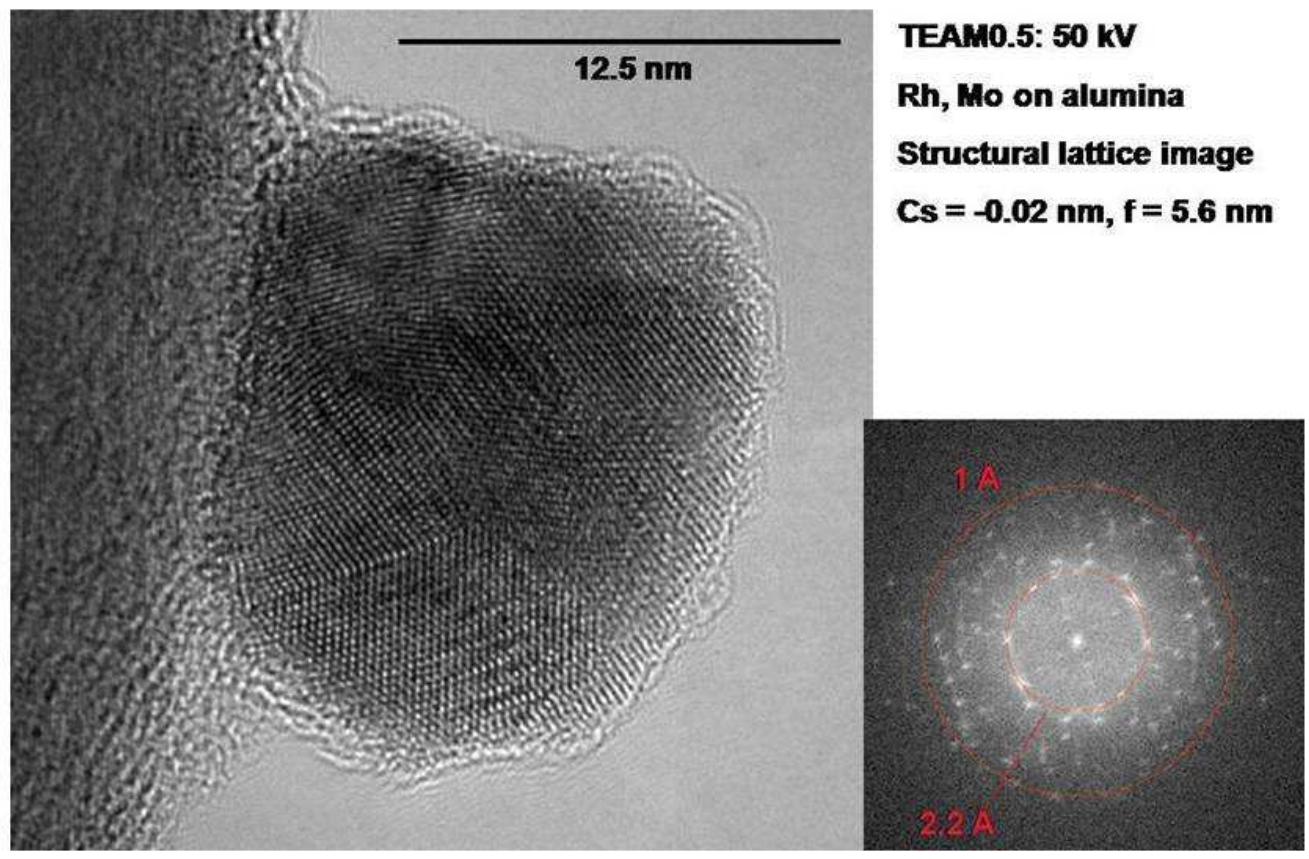

FIG. 1. Low voltage microscopy image of Rhodium particle on alumina support. Atomic structure and defects are clearly visible, the information limit (FFT, right side) extends beyond $1 \AA$. Acceleration voltage: $50 \mathrm{kV}$
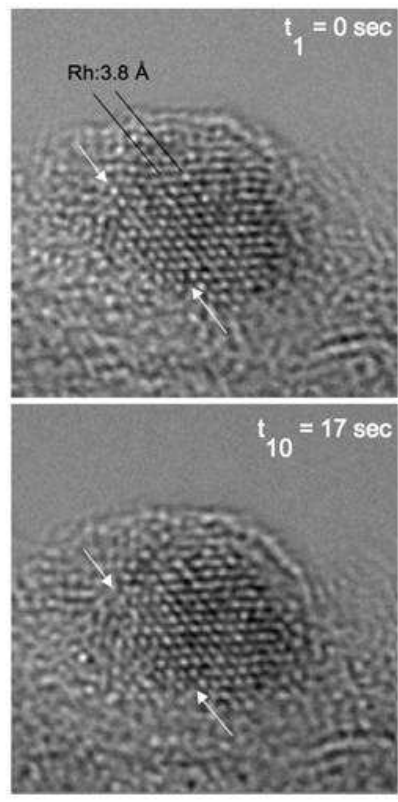
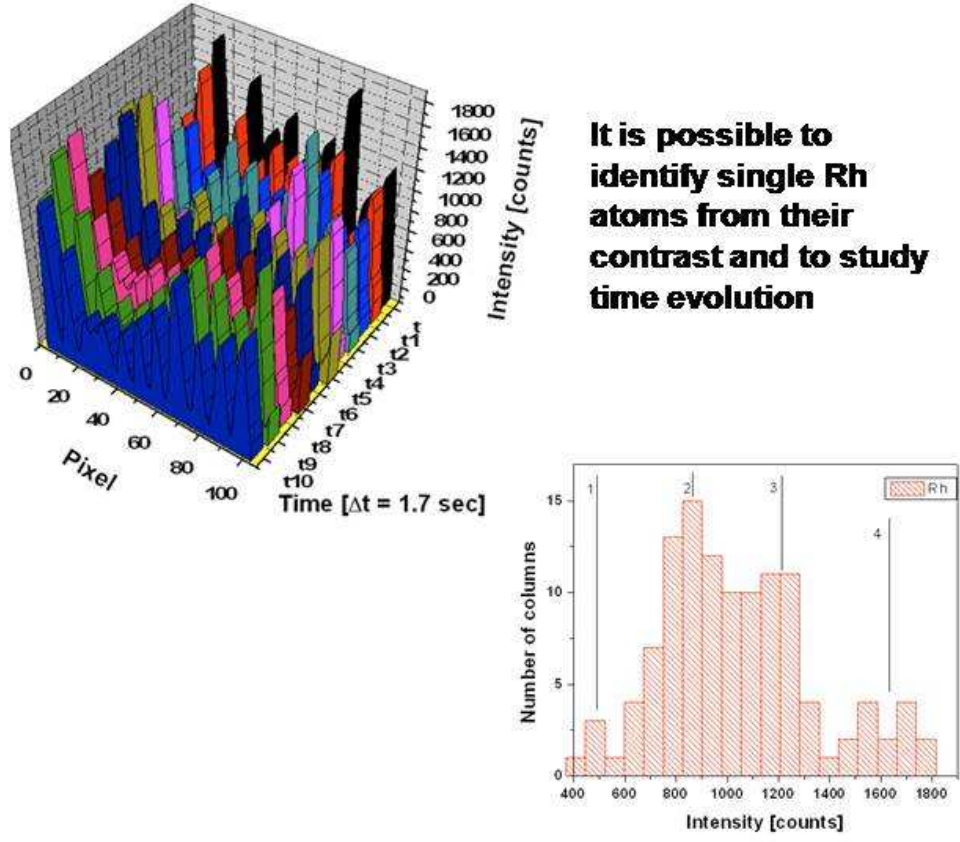

$1 \mathrm{Rh}$ atom $=420 \pm 130$ counts - contrast: $13 \pm 4 \%$

FIG. 2. Contrast evaluation of $\mathrm{Rh}$ particle on alumina support. Contrast values accumulate at multiples of 420 counts, as expected for one to four atoms per column for the applied image conditions. Motion of surface atoms can be observed in time series. Acceleration voltage: $80 \mathrm{kV}$ 